\title{
FDA Guidance on Assessment of Patient-Reported Outcomes in Cancer Trials: A Breath of Fresh Air or a Storm in a Teacup?
}

\author{
Ari Gnanasakthy ${ }^{1}$ (D) \\ Accepted: 21 July 2021 / Published online: 2 August 2021 \\ (c) The Author(s), under exclusive licence to Springer Nature Switzerland AG 2021
}

In June of this year, the US Food and Drug Administration (FDA) released a draft guidance to fill a much-needed gap related to formulating a strategy for the use of patientreported outcomes (PROs) in cancer trials [1]. Based on recognition of the diverse use of PRO measures employed in cancer trials, this draft guidance, which focuses on anticancer products, is an evolution of the PRO guidance released in 2009 [2]. It also further builds upon a previous FDA guidance that acknowledges the importance of PROs in cancer studies [3].

It is important to note that this latest draft guidance is not meant to be a road map to PRO labeling. Quite the opposite, in fact. Rather than offering a set of "FDA-approved" approaches to PRO assessment that guarantees labeling based on PRO-based endpoints, the latest guidance recommends core concepts to be assessed in cancer studies and underlines the merits of study-specific strategies. Moreover, and perhaps most importantly, the guidance signals reviewers' increased flexibility to entertain PRO instruments and approaches that best fit a study's need when considering the benefits and risks of new cancer therapies and highlights that the one-size-fits-all approach often historically used is not appropriate. Sponsors should no longer rely on inefficient strategies and fossilized instruments. Instead, the FDA's emphasis on the importance of using appropriate instruments and efficient assessment schedules opens new opportunities for sponsors to design trials that better reflect patient experience.

The 2021 draft guidance incorporates advice that the FDA has previously offered in separate discussions $[4,5]$. For this reason, readers versed in the PRO guidance of 2009 will recognize a few familiar threads. Consistent with the 2009 PRO guidance, there is a call for PRO-based endpoints to be well defined, valid, and reliable [1,6]. This

Ari Gnanasakthy

gnanasakthy@rti.org

1 RTI Health Solutions, Research Triangle Park, NC, USA is particularly important to emphasize because most PRO instruments used in cancer studies were developed prior to the 2009 PRO guidance and before the emergence of novel anticancer therapies. Recognizing this need, the agency offers flexibility for sponsors to demonstrate the appropriateness of legacy instruments, or appropriate components of legacy instruments, within the context of use based on prior experience or evidence from the literature.

Additionally, two primary aspects of formulating PRO strategies stand out as new contributions to this guidance. These address the types of assessments that FDA reviewers will consider and the timing of PRO data collection.

One of the major contributions of this latest guidance is the FDA's increased willingness to consider a la carte items or domains from established PRO item libraries (e.g., European Organisation for Research and Treatment of Cancer, PRO-Common Terminology Criteria for Adverse Events). This is a refreshing and much-needed acknowledgement from the agency that may resolve limitations arising from the use of legacy instruments, many of which contain items and/or concepts that may not be relevant to the condition or treatment and which, consequently, present an unnecessary burden on patients [4]. Patient burden is clearly at the forefront of the FDA's consideration: while admitting that there is no "magic number," the FDA suggests that PRO assessments should not take more than 10 min of the patient's time per visit [7], and they should measure what is relevant and important to the patient.

In concert with a reduction in the number of items administered to patients is the recommendation that PRO assessments be designed strategically to maximize the relevance of data while minimizing patient burden. The emphasis on frequent assessments of PROs during the early part of the study (while most patients are enrolled) with flexibility to stagger the assessment of different concepts at relevant assessment points is a pragmatic solution. This approach can lessen study and patient burden and meet the needs of multiple stakeholders without compromising scientific rigor. 
Notably, the FDA's prioritizing collection of data related to symptom experience during treatment cycles when the impact of the treatment is at its maximum (as often occurs with intravenous-based cancer treatments) eliminates the standard practice of collecting PRO data during a clinic visit on the first day of each treatment cycle. This helps offset difficulties caused by treatment delays and interruption during analysis and interpretation [8]. Furthermore, capturing data in a home setting makes patient convenience a priority and encourages electronic data capture.

Perhaps equally as important as the new contributions in this draft guidance are the aspects of the guidance that are not new. The discussion of statistical standards that are portrayed as key may look novel at first glance but are, in fact, as old as the hills. Rather than contributing to our understanding of new approaches, these sections perhaps offer the agency's insights on the shortcomings of the sponsor approach to the formulation of PRO strategies. Nowhere is this more evident than in the discussion of data integrity and analysis.

The issue of data quality described in the draft guidance is certainly well founded. Missing values, for example, represent a recognized problem in oncology trials [9-11]. Often, this is the result of inefficient procedures for data capture and monitoring and/or poor training for study staff and patients. Such issues may reflect the fact that PRO objectives (often vague) are frequently relegated to "exploratory" status in study protocols and, as a result, are not treated as a priority. The guidance stresses the need to establish procedures for mitigating missing data (including appropriate training for both investigators and patients) and the importance of documenting the reasons for missingness, advice that is rarely found in similar guidance documents from the FDA. The need to reestablish the basics of quality data capture may indicate that authors of guidance are frustrated (or unimpressed) with the quality of data currently submitted by study sponsors. While the extent of missing values is likely to be influenced by many factors, including the severity of cancer and treatment regimens, as a general rule, an $80 \%$ completion rate of PRO instruments at all timepoints up to 6 months from baseline is considered to be a minimum for anticancer trials [7].

Beyond these issues with data collection, problems with statistical analysis plans and data interpretation again force the draft guidance to package as revelatory an astoundingly basic statistical concept. The mistake: sponsors are quick to celebrate conclusions of "no difference" based on a mediocre analysis of data captured by insensitive instruments administered at irrelevant timepoints. Pathetic! To remedy this unfortunate practice, the draft guidance explicitly states that a lack of statistical superiority is not synonymous with "no meaningful difference." Rather, a claim of noninferiority or equivalence should be prespecified in the analysis plan and should be supported by evidence that the sensitivity of the instrument is adequate, that the study is adequately designed, and that the selected noninferiority margin is justified. Only time will tell if "equivalence" with respect to symptom severity or tolerability will ever be considered as a meaningful treatment benefit worthy of labeling.

The draft guidance certainly provides strong support for the spirit and execution of including PROs in cancer clinical trials, although practical oversights in the document may prevent it from being adopted wholesale by pharmaceutical companies. Chief among these is the guidance's implicit focus on randomized controlled trials, whereas most cancer clinical trials are open label. Of the 28 new anticancer drugs approved by the FDA in 2017 and 2018, only five included evidence from randomized double-blind controlled trials. From a regulatory standpoint, bias in open-label studies is a concern, and therefore PRO findings are unlikely to be included in product labeling [9].

If the open-label design of cancer trials represents one challenge for sponsors, the requirements of serving multiple stakeholders is another. Varying and sometimes contradictory requirements from the FDA, the European Medicines Agency (EMA), and other health technology assessment bodies may lead sponsors to feel overwhelmed by the prospect of incorporating PRO instruments into study protocols, particularly when such data are "nice to have" rather than essential [12]. Existing evidence supports this position: confirmatory studies of almost $30 \%$ of cancer indications approved by the FDA and EMA in 2012-16 did not include any PRO data [13]. Given these challenges (combined with the fact that PRO data are not mandatory for anticancer drug approvals), it is perhaps no wonder that integration of PROs in anticancer trial protocols is absent or suboptimal [12]. However, omission of PRO data can prevent benefit/risk assessments and be a source of frustration to reviewers, as indicated by one member of the Oncology Drug Advisory Committee (ODAC) during a recent review: “... I think it's come up multiple times before ODAC, where sponsors suggest that there is a better quality of life or benefit, but they do not have the data to back that up" [14].

Ultimately, the guidance offers a step forward and consolidated insight into the FDA's perspective, and it demonstrates the reviewers' willingness to acknowledge the challenges sponsors face in designing anticancer clinical trials. This, perhaps, is the real benefit of the FDA's 2021 draft guidance: by underlining the vital role PROs play in helping sponsors demonstrate treatment benefit, the agency has opened a window, airing out stuffy thinking on the value of patient input and breathing new life into patient-centric cancer clinical trials. 
Acknowledgements Sincere thanks to my colleague Lindsey Norcross of RTI Health Solutions for turning my random thoughts into sentences suitable for human comprehension and to my informed and experienced friends for their review of earlier drafts, often accompanied with appeals to restrain my derisive tendencies and consider softer, more winsome language. I thank them all for their suggestions and comments, while retaining full responsibility for the contents of this editorial.

\section{Declarations}

Funding No sources of funding were received for the preparation of this article.

Conflict of interest Ari Gnanasakthyhas no conflicts of interest that are directly relevant to the content of this article.

Ethics approval Not applicable.

Consent to participate Not applicable.

Consent for publication Not applicable.

Availability of data and material Not applicable.

Code availability Not applicable.

Authors' Contributions Ari Gnanasakthyhas is the sole author of this article.

\section{References}

1. US Department of Health and Human Services, Food and Drug Administration, Center for Drug Evaluation and Research, Center for Biologics Evaluation and Research, Center for Devices and Radiological Health. Guidance for industry: core patient-reported outcomes in cancer clinical trials. 2021. https://www.fda.gov/ media/149994/download. Accessed 24 Jul 2021.

2. US Department of Health and Human Services, Food and Drug Administration, Center for Drug Evaluation and Research, Center for Biologics Evaluation and Research, \& Center for Devices and Radiological Health. Guidance for industry: patient-reported outcomes measures: use in medical product development to support labeling claims. 2009. https://www.fda.gov/media/77832/downl oad. Accessed 26 July 2021.

3. US Department of Health and Human Services, Food and Drug Administration, Center for Drug Evaluation and Research, Center for Biologics Evaluation and Research, \& Center for Devices and Radiological Health. Guidance for industry: clinical trial endpoints for the approval of cancer drugs and biologics. 2018. https://www.fda.gov/media/71195/download. Accessed 24 Jul 2021.

4. Kluetz PG, Slagle A, Papadopoulos EJ, Johnson LL, Donoghue M, Kwitkowski VE, et al. Focusing on core patient-reported outcomes in cancer clinical trials: symptomatic adverse events, physical function, and disease-related symptoms. Clin Cancer Res. 2016;22(7):1553-8.

5. Kluetz PG, Kanapuru B, Lemery S, Johnson LL, Fiero MH, Arscott $\mathrm{K}$, et al. Informing the tolerability of cancer treatments using patient-reported outcome measures: summary of an FDA and critical path institute workshop. Value Health. 2018;21(6):742-7.

6. Walton MK, Powers JH 3rd, Hobart J, Patrick D, Marquis P, Vamvakas $\mathrm{S}$, et al. Clinical outcome assessments: conceptual foundation-report of the ISPOR Clinical Outcomes Assessment-Emerging Good Practices for Outcomes Research Task Force. Value Health. 2015;18(6):741-52.

7. Sutter S. Patient-reported outcomes in pediatric cancer trials endorsed by the US FDA Advisory Committee. Pink Sheet. 14 May 2021.

8. Chirila C, Odom D, Gnanasakthy A. Patient-reported outcomes assessment scheduling in oncology: are we introducing potential bias? DIA 2021 Global Annual Meeting; June 27.

9. Roydhouse JK, Mishra-Kalyani PS, Bhatnagar V, Gutman R, King-Kallimanis BL, Sridhara R, et al. Does knowledge of treatment assignment affect patient report of symptoms, function, and health status? An evaluation using multiple myeloma trials. Value Health. 2021;24(6):822-9.

10. Lombardi P, Marandino L, De Luca E, Zichi C, Reale ML, Pignataro D, et al. Quality of life assessment and reporting in colorectal cancer: a systematic review of phase III trials published between 2012 and 2018. Crit Rev Oncol Hematol. 2020;146:102877.

11. Wilson MK, Friedlander ML, Joly F, Oza AM. A systematic review of health-related quality of life reporting in ovarian cancer phase III clinical trials: room to improve. Oncologist. 2018;23(2):203-13.

12. Retzer A, Calvert M, Ahmed K, Keeley T, Armes J, Brown JM, et al. International perspectives on suboptimal patient-reported outcome trial design and reporting in cancer clinical trials: a qualitative study. Cancer Med. 2021;1-13. https://doi.org/10.1002/ cam4.4111

13. Gnanasakthy A, Barrett A, Evans E, D'Alessio D, Romano C. A review of patient-reported outcomes labeling for oncology drugs approved by the FDA and the EMA (2012-2016). Value Health. 2019;22(2):203-9.

14. US Food and Drug Administration. Oncology Drug Advisory Committee Meeting (transcript December 18, 2019). 2019. https:// www.fda.gov/media/139805/download. Accessed 24 Jul 2021. 